\title{
Hybrid TSR-PSR in nonlinear EH half duplex network: system performance analysis
}

\author{
Phu Tran Tin ${ }^{1}$, Duy-Hung $\mathrm{Ha}^{2}$, Tran Thanh Trang ${ }^{3}$ \\ ${ }^{1}$ Faculty of Electronics Technology, Industrial University of Ho Chi Minh City, Ho Chi Minh City, Vietnam \\ ${ }^{2}$ Wireless Communications Research Group, Faculty of Electrical and Electronics Engineering, \\ Ton Duc Thang University, Ho Chi Minh City, Vietnam \\ ${ }^{3}$ National Key Laboratory of Digital Control and System Engineering, Ho Chi Minh City, Vietnam
}

\begin{tabular}{l} 
Article Info \\
\hline Article history: \\
Received May 10, 2019 \\
Revised Oct 9, 2019 \\
Accepted Oct 15, 2019 \\
\hline
\end{tabular}

\section{Keywords:}

Energy harvesting (EH)

Half-duplex (HD)

Monte Carlo simulation

Relaying network

Success probability (SP)

\begin{abstract}
Nowadays, harvesting energy (EH) from green environmental sources and converting this energy into the electrical energy used in purpose to supply the communication network devices is considered the main research direction. In this research, we investigate the hybrid TSR-PSR Nonlinear Energy Harvesting (EH) Half-duplex (HD) Relaying network in terms of the Success Probability (SP). For this purpose, we derive the integral-form of the system SP. In addition, we use the Monte Carlo simulation for verifying the correctness of the analytical expression. We can see in the research results that all the simulation and analytical values are the same in connection with all primary system parameters.
\end{abstract}

Copyright $\odot 2020$ Institute of Advanced Engineering and Science. All rights reserved.

\section{Corresponding Author:}

Duy-Hung Ha,

Wireless Communications Research Group,

Faculty of Electrical and Electronics Engineering,

Ton Duc Thang University,

Ho Chi Minh City, Vietnam.

Email: haduyhung@tdtu.edu.vn

\section{INTRODUCTION}

Nowadays, harvesting energy (EH) from green environmental sources and converting this energy into the electrical energy used in purpose to supply the communication network devices is considered the main research direction. Furthermore, this solution can provide not only the environmentally friendly energy supplies, but also the self-maintained, long-lived, and autonomous communication systems. In the series of the leading environmental green energy sources, such as solar, wind, geothermal, and mechanical, the radio frequency (RF) signals can be considered as the prospective energy source in the future. [1-9]. Wireless nodes can harvest RF energy either in the time domain before data reception, or in the power domain by dividing the received RF signals for the EH and information decoding (ID) [9-12]. In a cooperative network, authors in [13-16] developed two new relaying protocols based on the receiver structures adopted at R, termed time switching-based relaying (TSR) and power splitting-based relaying (PSR). From [14-16], the TSR and PSR protocols have some drawbacks; for instance, TSR has to lose some information while it switches to the harvesting mode and PSR has a low coverage area. In another way, PSR requires a complicated hardware structure to make sure that a proper portion of energy from the source signal is extracted for energy harvesting. In contrast, TSR can simplify the hardware at the expense of the throughput or achievable rate of the system. Based on the fact that both TSR and PSR protocols have their drawbacks, the prospective idea is to combine these two protocols to get the best out of them. This is a solution that can obtain in this paper by using an adaptive relaying protocol [17-18]. 
In this research, we investigate the hybrid TDR-PSR Nonlinear Energy Harvesting (EH) Halfduplex (HD) Relaying network in terms of the Success Probability (SP). For this purpose, we derive the integral form of the SP in connection with all primary system parameters. Also, we use the Monte Carlo simulation for verifying the correctness of the analytical expression. We can focus on the main contributions as the follows

a. The hybrid TDR-PSR Nonlinear EH HD Relaying network is proposed and investigated.

b. The integral-form expressions of the system SP are derived.

c. The correctness of the analytical expressions are verified by the Monte Carlo simulation.

\section{SYSTEM MODEL}

In this paper, the hybrid TDR-PSR Nonlinear EH HD Relaying network is illustrated in Figure 1. In this model, the information is transferred from the source $(S)$ to the multi-destination $\left(\mathrm{D}_{\mathrm{i}}\right)$, through relay node $(\mathrm{R})$. The energy harvesting $(\mathrm{EH})$ and information transmission (IT) of the system model are proposed in Figure 2. As shown in Figure 2, $\mathrm{T}$ is the block time. In the first interval time $(\alpha \mathrm{T})$, the relay node $\mathrm{R}$ harvests energy $\rho P_{s}$ and receives the information $(1-\rho) P_{s}$ from the source signal, where $\alpha$ is the time switching factor $\alpha \in(0,1)$ and $\rho$ is the power splitting factor $\rho \in(0,1)$. In the remaining interval time $(1-\alpha) \mathrm{T}$, the relay node $\mathrm{R}$ transfers information to the destination node $\mathrm{D}$ [12-16].

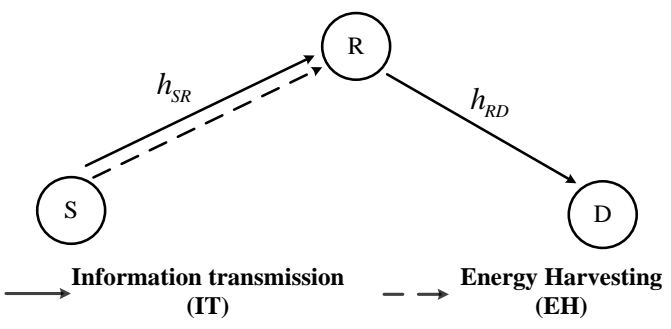

Figure 1. System model

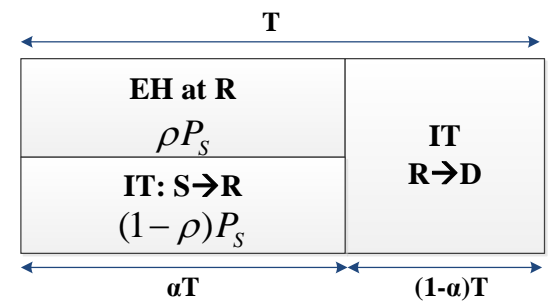

Figure 2. The EH and ITphases

\subsection{Energy harvesting (EH) phase}

In this phase, the received signal at the relay $\mathrm{R}$ can be given as

$$
y_{r}=\sqrt{\rho P_{s}} h_{S R} x_{s}+n_{r}
$$

Where $x_{s}$ is the energy symbol and must be satisfied $\mathrm{E}\left\{\left|x_{s}\right|^{2}\right\}=1$ which $\mathrm{E}\{\bullet\}$ is the expectation operator.

$\mathrm{P}_{\mathrm{s}}$ is the transmit power of the source.

$\mathrm{n}_{\mathrm{r}}$ is the additive white Gaussian noise (AWGN) at the relay node with variance $\mathrm{N}_{0}$.

$h_{S R}$ is channel gain between S-R link and belongs to Rayleigh channel.

In this paper, the nonlinear transformation model proposed in reference [19] is used. The transmission power at the relay can be given as

$$
P_{r}=\left[\begin{array}{ll}
\kappa P_{s}\left|h_{S R}\right|^{2}, P_{s}\left|h_{S R}\right|^{2} \leq P_{t h} \\
\kappa P_{t h}, & P_{s}\left|h_{S R}\right|^{2}>P_{t h}
\end{array}\right.
$$


Where we denote $\kappa=\frac{\eta \rho \alpha}{1-\alpha}, P_{t h}$ is the saturation threshold of the rechargeable power of the hardware circuit.

\subsection{Information transmission (IT) phase}

The received signal at the relay $\mathrm{R}$ in the first time slot can be calculated as

$$
y_{r}=\sqrt{(1-\rho) P_{s}} h_{S R} x_{s}+n_{r}
$$

In the second time slot, after receiving the signal from the source $\mathrm{R}$, the relay amplifíes with $\beta$ factor as following

$$
\beta=\frac{x_{r}}{y_{r}}=\sqrt{\frac{1}{(1-\rho) P_{s}\left|h_{S R}\right|^{2}+N_{0}}}
$$

The received signal at the destination $\mathrm{D}$ can be formulated as

$$
y_{d}=\sqrt{P_{r}} h_{R D} x_{r}+n_{d}
$$

Where $x_{r}$ is the transmission signal at relay and must be satisfied $\mathrm{E}\left\{\left|x_{r}\right|^{2}\right\}=1, \mathrm{n}_{\mathrm{d}}$ is the additive white Gaussian noise (AWGN) at the relay node with variance $\mathrm{N}_{0}, h_{R D}$ is channel gain between R-D link and also belongs to Rayleigh channel.

Substituting (4) into (5) and then combine with (3), (5) can be rewritten as

$$
\begin{aligned}
y_{d} & =\sqrt{P_{r}} h_{R D} \beta y_{r}+n_{d}=\sqrt{P_{r}} h_{R D} \beta\left[\sqrt{(1-\rho) P_{s}} h_{S R} x_{s}+n_{r}\right]+n_{d} \\
& =\underbrace{\sqrt{(1-\rho) P_{r}} h_{S R} x_{s} \sqrt{P_{r}} h_{R D} \beta}_{\text {signal }}+\underbrace{\sqrt{P_{r}} h_{R D} \beta n_{r}+n_{d}}_{\text {noise }}
\end{aligned}
$$

The end to end signal to noise ratio can be computed as

$$
\begin{aligned}
\gamma_{e 2 e} & =\frac{(1-\rho) P_{r} P_{s}\left|h_{S R}\right|^{2}\left|h_{R D}\right|^{2} \beta^{2}}{P_{r}\left|h_{R D}\right|^{2} \beta^{2} N_{0}+N_{0}}=\frac{(1-\rho) P_{r} P_{s}\left|h_{S R}\right|^{2}\left|h_{R D}\right|^{2}}{P_{r}\left|h_{R D}\right|^{2} N_{0}+N_{0}\left[(1-\rho) P_{s}\left|h_{S R}\right|^{2}+N_{0}\right]} \\
& \approx \frac{(1-\rho) P_{s}\left|h_{S R}\right|^{2}\left|h_{R D}\right|^{2}}{\left|h_{R D}\right|^{2} N_{0}+\frac{(1-\rho) P_{s}\left|h_{S R}\right|^{2} N_{0}}{P_{r}}}
\end{aligned}
$$

\section{THE SYSTEM PERFORMANCE}

The successful probability (SP) can be defined as

$$
S P=\operatorname{Pr}\left(\gamma_{e 2 e}>\gamma_{t h}\right)=\operatorname{Pr}\left(\frac{(1-\rho) P_{s}\left|h_{S R}\right|^{2}\left|h_{R D}\right|^{2}}{\left|h_{R D}\right|^{2} N_{0}+\frac{(1-\rho) P_{s}\left|h_{S R}\right|^{2} N_{0}}{P_{r}}}>\gamma_{t h}\right)
$$

Where $\gamma_{t h}$ is the threshold of the system. 
Substituting (2) into (8), (8) can be obtained as

$$
\begin{aligned}
S P= & \operatorname{Pr}\left(\frac{(1-\rho) P_{s}\left|h_{S R}\right|^{2}\left|h_{R D}\right|^{2}}{\left|h_{R D}\right|^{2} N_{0}+\frac{(1-\rho) N_{0}}{\kappa}}>\gamma_{t h}, P_{s}\left|h_{S R}\right|^{2} \leq P_{t h}\right) \\
& +\operatorname{Pr}\left(\frac{(1-\rho) P_{s}\left|h_{S R}\right|^{2}\left|h_{R D}\right|^{2}}{\left|h_{R D}\right|^{2} N_{0}+\frac{(1-\rho) P_{s}\left|h_{S R}\right|^{2} N_{0}}{\kappa P_{t h}}}>\gamma_{t h}, P_{s}\left|h_{S R}\right|^{2}>P_{t h}\right) \\
& =P_{1}+P_{2}
\end{aligned}
$$

Where we denote

$$
P_{1}=\operatorname{Pr}\left(\frac{(1-\rho) P_{s}\left|h_{S R}\right|^{2}\left|h_{R D}\right|^{2}}{\left|h_{R D}\right|^{2} N_{0}+\frac{(1-\rho) N_{0}}{\kappa}}>\gamma_{t h}, P_{s}\left|h_{S R}\right|^{2} \leq P_{t h}\right)
$$

We denote

$$
X=\left|h_{S R}\right|^{2}, Y=\left|h_{R D}\right|^{2} \text { and } \Psi=\frac{P_{s}}{N_{0}}, \chi=\frac{P_{t h}}{P_{s}} .
$$

The (10) can be rewritten as

$$
P_{1}=\operatorname{Pr}\left(\frac{(1-\rho) \Psi X Y}{Y+\frac{(1-\rho)}{\kappa}}>\gamma_{t h}, X \leq \chi\right)=\int_{0}^{\chi} \underbrace{\operatorname{Pr}\left[\frac{(1-\rho) \Psi x Y}{Y+\frac{(1-\rho)}{\kappa}}>\gamma_{t h}\right]}_{\Phi(x)} f_{X}(x) d x
$$

Here, considering that

$$
\begin{aligned}
\Phi(x) & =\operatorname{Pr}\left[\frac{(1-\rho) \Psi x Y}{Y+\frac{(1-\rho)}{\kappa}}>\gamma_{t h}\right]=\operatorname{Pr}\left[Y\left\{(1-\rho) \Psi x-\gamma_{t h}\right\}>\frac{(1-\rho) \gamma_{t h}}{\kappa}\right] \\
& = \begin{cases}\operatorname{Pr}\left[Y>\frac{(1-\rho) \gamma_{t h}}{\kappa\left\{(1-\rho) \Psi x-\gamma_{t h}\right\}}\right], & x \geq \frac{\gamma_{t h}}{(1-\rho) \Psi} \\
0 \quad, & x<\frac{\gamma_{t h}}{(1-\rho) \Psi}\end{cases} \\
& = \begin{cases}\exp \left[-\frac{(1-\rho) \lambda_{r d} \gamma_{t h}}{\kappa\left\{(1-\rho) \Psi x-\gamma_{t h}\right\}}\right], & x \geq \frac{\gamma_{t h}}{(1-\rho) \Psi} \\
0 \quad & , x<\frac{\gamma_{t h}}{(1-\rho) \Psi}\end{cases}
\end{aligned}
$$

Where $\lambda_{r d}$ is the mean value of the random variable (RV) $\left|h_{R D}\right|^{2}$. 
We assume that $\frac{\gamma_{\text {th }}}{(1-\rho) \Psi} \leq \chi$ and then substituting (12) into (11), we have

$$
P_{1}=\lambda_{s r} \int_{\frac{\gamma_{t h}}{(1-\rho) \Psi}}^{\chi} \exp \left[-\frac{(1-\rho) \lambda_{r d} \gamma_{t h}}{\kappa\left\{(1-\rho) \Psi x-\gamma_{t h}\right\}}\right] \times \exp \left(-\lambda_{s r} x\right) d x
$$

Now, we will find $\mathrm{P}_{2}$ from (9) as the following

$$
P_{2}=\operatorname{Pr}\left(\frac{(1-\rho) \Psi X Y}{Y+\frac{(1-\rho) X}{\kappa \chi}}>\gamma_{t h}, X>\chi\right)=\int_{\chi}^{\infty} \operatorname{Pr}\left[\frac{(1-\rho) \Psi x Y}{Y+\frac{(1-\rho) x}{\kappa \chi}}>\gamma_{t h}\right] f_{X}(x) d x
$$

Where

$$
\begin{aligned}
\Theta(x) & =\operatorname{Pr}\left[\frac{(1-\rho) \Psi x Y}{Y+\frac{(1-\rho) x}{\kappa \chi}}>\gamma_{t h}\right]=\operatorname{Pr}\left\{Y\left[(1-\rho) \Psi x-\gamma_{t h}\right]>\frac{(1-\rho) x \gamma_{t h}}{\kappa \chi}\right\} \\
& = \begin{cases}\operatorname{Pr}\left\{Y>\frac{(1-\rho) x \gamma_{t h}}{\kappa \chi\left[(1-\rho) \Psi x-\gamma_{t h}\right]}\right\}, & x \geq \frac{\gamma_{t h}}{(1-\rho) \Psi} \\
0 & , x<\frac{\gamma_{t h}}{(1-\rho) \Psi} \\
\exp \left\{-\frac{(1-\rho) \lambda_{r d} x \gamma_{t h}}{\kappa \chi\left[(1-\rho) \Psi x-\gamma_{t h}\right]}\right\}, & x \geq \frac{\gamma_{t h}}{(1-\rho) \Psi} \\
0 & , x<\frac{\gamma_{t h}}{(1-\rho) \Psi}\end{cases}
\end{aligned}
$$

Substituting (15) into (14), $\mathrm{P}_{2}$ can be claimed as

$$
P_{2}=\lambda_{s r} \int_{\chi}^{\infty} \exp \left\{-\frac{(1-\rho) \lambda_{r d} x \gamma_{t h}}{\kappa \chi\left[(1-\rho) \Psi x-\gamma_{t h}\right]}\right\} \times \exp \left(-\lambda_{s r} x\right) d x
$$

Finally, we can obtain the SP as following

$$
\begin{aligned}
S P & =\lambda_{s r} \int_{\frac{\gamma_{t h}}{(1-\rho) \Psi}}^{\chi} \exp \left[-\frac{(1-\rho) \lambda_{r d} \gamma_{t h}}{\kappa\left\{(1-\rho) \Psi x-\gamma_{t h}\right\}}\right] \times \exp \left(-\lambda_{s r} x\right) d x \\
& +\lambda_{s r} \int_{\chi}^{\infty} \exp \left\{-\frac{(1-\rho) \lambda_{r d} x \gamma_{t h}}{\kappa \chi\left[(1-\rho) \Psi x-\gamma_{t h}\right]}\right\} \times \exp \left(-\lambda_{s r} x\right) d x
\end{aligned}
$$

\section{NUMERICAL RESULTS AND DISCUSSION}

In this section, the Monte Carlo simulation is used for validating the analytical expression in the above section [20-25]. Figure 3 shows the SC versus time switching factor $\alpha$ with the main system parameters as $\eta=0.4,0.85 ; \psi=\mathrm{P}_{\mathrm{th}}=5 \mathrm{~dB} ; \rho=0.5$ and $\gamma_{\mathrm{th}}=0.25$. From Figure 3 we can state that the SP of the system network has a massive increase while time switching factor $\alpha$ varies from 0 to 0.9 , and the simulation and analytical values are the same. Moreover, the effect of energy efficiency $\eta$ on the system SP. Here, we set $\alpha=0.5 ; \rho=0.5$ and $\gamma_{\mathrm{th}}=0.25$. Similarity as Figure 4 , the system SP increases significantly with the rising of energy efficiency $\eta$, and the analytical results agree with the simulation values. 
Furthermore, the influence of $\mathrm{P}_{\text {th }}$ and $\psi$ on the system SP are drawn in Figures 5 and 6, respectively. From the results, we can see that the system SP increases considerably with the rising of $\mathrm{P}_{\text {th }}$ and $\psi$. In addition, the system SP versus the power splitting factor $\rho$ is presented in Figure 7. From Figure 7, we can see that the system SP has a considerable increase in the first interval of $\rho$ and the has a decease. The optimal value of the system SP can be obtained with the values of $\rho$ from 0.5 to 0.6 . In all Figures 5-7, the simulation values match well with the analytical values for verifying the correctness of the analytical expressions.

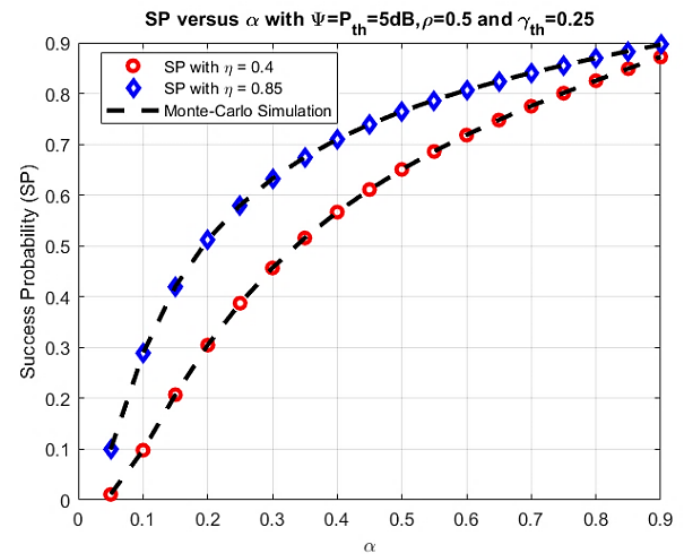

Figure 3. SP versus $\alpha$

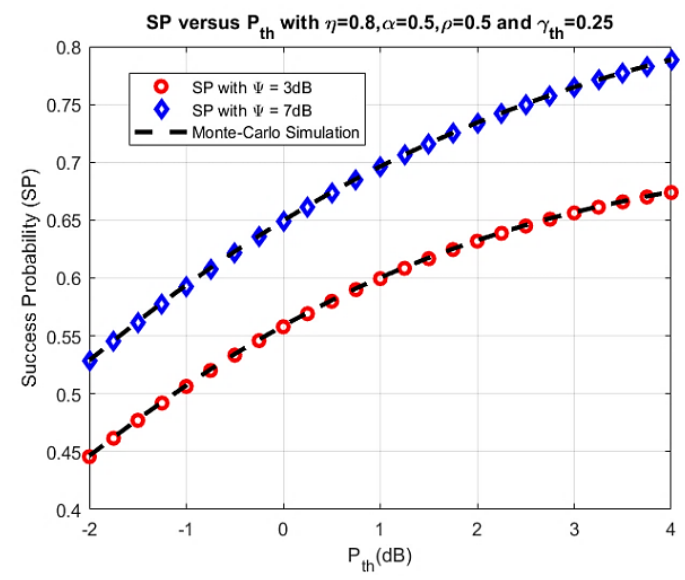

Figure 5. SP versus $\mathrm{P}_{\text {th }}$

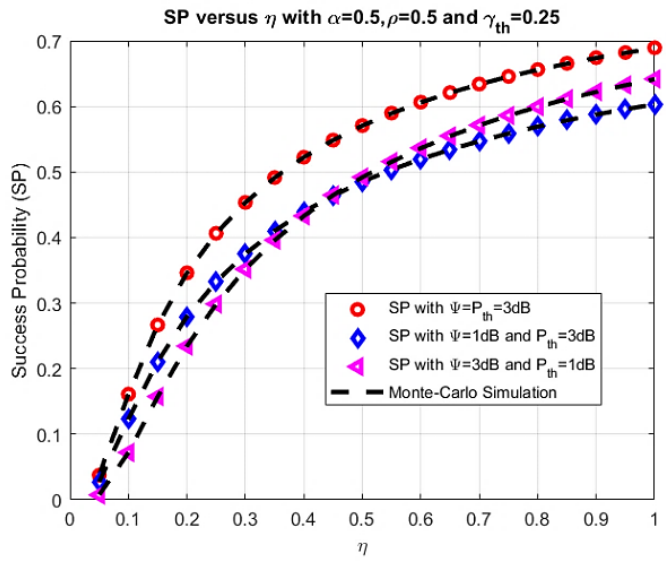

Figure 4. SP versus $\eta$

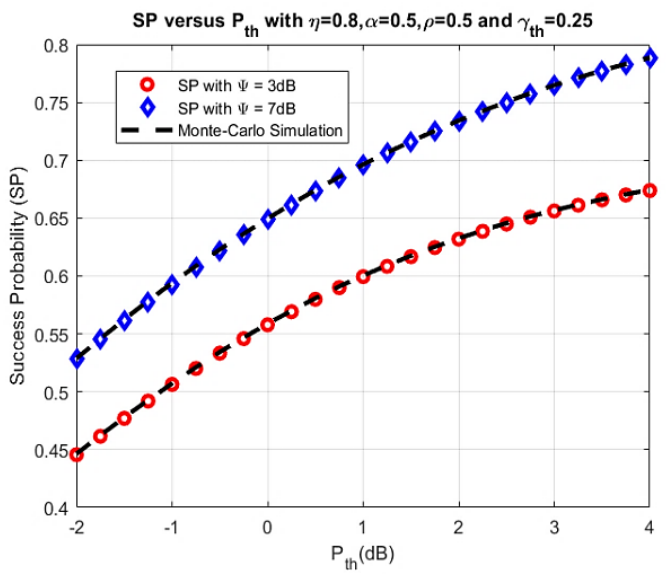

Figure 6. SP versus $\psi$

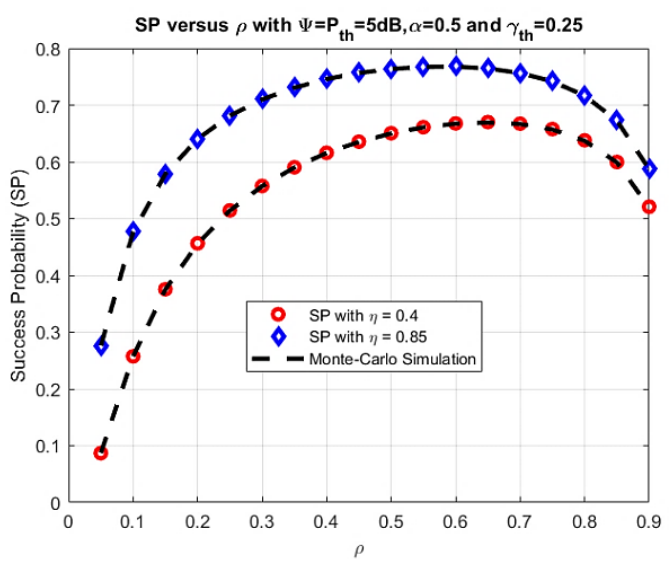

Figure 7. SP versus $\rho$ 


\section{CONCLUSION}

In this research, we investigate the hybrid TDR-PSR Nonlinear Energy EH HD Relaying network in terms of the SP. Firstly, we derive the integral form of the SP in connection with all primary system parameters. In addition, we use the Monte Carlo simulation for verifying the correctness of the analytical expression. From the research results, we can state that all the simulation and analytical values are the same in connection with all primary system parameters.

\section{ACKNOWLEDGMENTS}

This research was supported by National Key Laboratory of Digital Control and System Engineering (DCSELAB), HCMUT, VNU-HCM, Vietnam.

\section{REFERENCES}

[1] Bi, S., Ho, C. K., \& Zhang, R., "Wireless powered communication: Opportunities and challenges," IEEE Communications Magazine, vol. 53, no. 4, pp. 117-125, 2015. doi:10.1109/mcom.2015.7081084

[2] Niyato, D., Kim, D. I., Maso, M., and Han, Z., "Wireless powered communication networks: Research directions and technological approaches," IEEE Wireless Communications, pp. 2-11, 2017. doi:10.1109/mwc.2017.1600116

[3] Yu, H., Lee, H., \& Jeon, H., "What is 5G? Emerging 5G mobile services and network requirements," Sustainability, vol. 9, no. 10, pp. 1848, 2017. doi:10.3390/su9101848.

[4] Zhou, Xun, Rui Zhang, and Chin Keong Ho., "Wireless information and power transfer: Architecture design and rate-energy tradeoff," IEEE Transactions on Communications, vol. 61, no. 11, pp. 4754-767, 2013. doi:10.1109/tcomm.2013.13.120855.

[5] Akhtar, Rizwan, Supeng Leng, Imran Memon, Mushtaq Ali, and Liren Zhang, "Architecture of hybrid mobile social networks for efficient content delivery," Wireless Personal Communications, vol. 80, pp. 85-96, 2014. doi:10.1007/s11277-014-1996-4.

[6] Rango, Floriano De, Mario Gerla, and Salvatore Marano, "A scalable routing scheme with group motion support in large and dense wireless ad hoc networks," Computers \& Electrical Engineering, vol. 32, pp. 224-40, 2006. doi:10.1016/j.compeleceng.2006.01.017.

[7] Zhou, Biao, Yeng-Zhong Lee, Mario Gerla, and Floriano De Rango, "Geo-LANMAR: A scalable routing protocol for ad hoc networks with group motion," Wireless Communications and Mobile Computing, vol. 6, no. 7, pp. 989-1002, 2006. doi:10.1002/wcm.433.

[8] Fazio, Peppino, Floriano De Rango, Cesare Sottile, and Carlos Calafate, "A new channel assignment scheme for interference-aware routing in vehicular networks," 2011 IEEE $73^{\text {rd }}$ Vehicular Technology Conference (VTC Spring), 2011. doi:10.1109/vetecs.2011.5956777.

[9] Cassano, E., F. Florio, F. De Rango, and S. Marano, "A performance comparison between ROC-RSSI and trilateration localization techniques for WPAN sensor networks in a real outdoor testbed," 2009 Wireless Telecommunications Symposium, 2009. doi:10.1109/wts.2009.5068988.

[10] Chen, He, Chao Zhai, Yonghui $\mathrm{Li}$, and Branka Vucetic, "Cooperative strategies for wirelesspowered communications: an overview," IEEE Wireless Communications, vol, 25, no. 4, pp. 112-19, 2018. doi:10.1109/mwc.2017.1700245.

[11] Zhai, Chao, Ju Liu, and Lina Zheng, "Relay-based spectrum sharing with secondary users powered by wireless energy harvesting," IEEE Transactions on Communications, vol. 64, no. 5, pp. 1875-887, 2016. doi:10.1109/tcomm.2016.2542822.

[12] Zhai, Chao, Lina Zheng, Peng Lan, He Chen, and Hongi Xu, "Decode-and-forward two-path successive relaying with wireless energy harvesting," 2017 IEEE International Conference on Communications Workshops (ICC Workshops), 2017. doi:10.1109/iccw.2017.7962630.

[13] Chen, Yunfei, R. Shi, Wei Feng, and Ning Ge, "AF relaying with energy harvesting source and relay," IEEE Transactions on Vehicular Technology, 2016. doi:10.1109/tvt.2016.2556639.

[14] Zheng, Lina, Chao Zhai, and Ju Liu, "Alternate energy harvesting and information relaying in cooperative AF networks," Telecommunication Systems, vol. 68, no. 3, pp. 523-33, 2017. doi:10.1007/s11235-017-0399-8.

[15] Abbas, Hatem, and Khairi Hamdi, "Millimeter wave communications over relay networks," 2017 IEEE Wireless Communications and Networking Conference (WCNC), 2017. doi:10.1109/wcnc.2017.7925836.

[16] Kong, Linghe, Muhammad Khurram Khan, Fan Wu, Guihai Chen, and Peng Zeng, "Millimeter-wave wireless communications for IoT-cloud supported autonomous vehicles: Overview, design, and challenges," IEEE Communications Magazine, vol. 55, no. 1, pp. 62-68, 2017. doi:10.1109/mcom.2017.1600422cm.

[17] Zhou, Zheng, Mugen Peng, Zhongyuan Zhao, and Yong Li, "Joint power splitting and antenna selection in energy harvesting relay channels," IEEE Signal Processing Letters, vol. 22, no. 7, pp. 823-27, 2015. doi:10.1109/lsp.2014.2369748.

[18] Liu, L., Zhang, R., and Chua, K., "Wireless information and power transfer: A dynamic power splitting approach," IEEE Transactions on Communications, vol. 61(9), pp. 3990-4001, 2013. doi:10.1109/tcomm.2013.071813.130105

[19] Y. Dong, M. J. Hossain and J. Cheng, "Performance of wireless powered amplify and forward relaying over nakagami-m fading channels with nonlinear energy harvester," IEEE Communications Letters, vol. 20, no. 4, pp. 672-675, 2016. 
[20] Tin, Phu Tran, Tran Hoang Quang Minh, Tan N. Nguyen, and Miroslav Voznak, "System performance analysis of half-duplex relay network over rician fading channel," TELKOMNIKA (Telecommunication, Computing, Electronics and Control), vol. 16, no. 1, pp. 189, 2018. doi:10.12928/telkomnika.v16i1.7491.

[21] Rashid, Tarique, Sunil Kumar, Akshay Verma, Prateek Raj Gautam, and Arvind Kumar, "Pm-EEMRP: Postural movement based energy efficient multi-hop routing protocol for intra wireless body sensor network (IntraWBSN)," TELKOMNIKA (Telecommunication, Computing, Electronics and Control), vol. 16, no. 1, pp. 166, 2018. doi:10.12928/telkomnika.v16i1.7318.

[22] A. F. Morabito, "Power synthesis of mask-constrained shaped beams through maximally-sparse planar arrays," TELKOMNIKA (Telecommunication, Computing, Electronics and Control), vol. 14, no. 4, pp. 1217-1219, 2016.

[23] Nguyen, T., Quang Minh, T., Tran, P., Vozňák, M., "Energy harvesting over rician fading channel: A performance analysis for half-duplex bidirectional sensor networks under hardware impairments," Sensors, vol. 18, pp. 1781, 2018.

[24] Tan N. Nguyen, T.H.Q. Minh, Phuong T. Tran, Miroslav Voznak, T.T. Duy, Thanh-Long Nguyen and Phu Tran Tin, "Performance enhancement for energy harvesting based two-way relay protocols in wireless ad-hoc networks with partial and full relay selection methods," Ad hoc networks, 2019.

[25] Nguyen, T.N., Minh, T.H.Q., Nguyen, T.-L., Ha, D.-H., Voznak, M., "Performance analysis of user selection protocol in cooperative networks with power splitting protocol based energy harvesting over nakagami-m/rayleigh channel," Electronics, vol. 8, p. 448, 2019. 